\title{
Relação entre o estado nutricional, comportamento alimentar e satisfação corporal de escolares adolescentes de Santa Cruz do Sul, RS
}

\author{
Relationship between nutritional status, body image perception and \\ eating behavior of schoolchildren from Santa Cruz do Sul, RS
}

\author{
Camila Caroline dos Santos, ${ }^{1}$ Fabiana Assmann Poll, ${ }^{1}$ Patrícia Molz² \\ Universidade de Santa Cruz do Sul (Unisc), Santa Cruz do Sul, RS, Brasil. \\ ${ }^{2}$ Pontificia Universidade Católica do Rio Grande do Sul, Porto Alegre, RS, Brasil.
}

Recebido em: 26/07/2016 / Aceito em: 22/08/2016 / Publicado em: 18/10/2016 fpol/@unisc.br

\section{RESUMO}

Objetivo: relacionar o estado nutricional, comportamento alimentar e a satisfação corporal de escolares adolescentes de Santa Cruz do Sul, RS. Método: trata-se de um estudo transversal, realizado com 54 escolares adolescentes com idades entre 10 até 12 anos $(10,49 \pm 0,50$ anos). O estado nutricional foi avaliado por meio do cálculo do Índice de Massa Corporal (IMC) e o comportamento alimentar foi avaliado pelo questionário Eating Behaviours and Body Image Test (EBBIT). Para a avaliação da satisfação com a imagem corporal, utilizou-se a escala de silhuetas proposta por Tiggeman e Wilson-Barrett. Resultados: do total de 54 escolares adolescentes estudados, a maioria apresentou eutrofia $(53,7 \%)$ e $61,1 \%$ estavam insatisfeitos para o excesso de peso. Verificou-se uma associação significativa entre a insatisfação com a imagem corporal e o estado nutricional $(p=0,001)$. Observou-se também diferença significativa entre o estado nutricional e no somatório dos fatores do teste EBBIT $(p<0,001)$, bem como com a pontuação do somatório da satisfação com a imagem corporal $(p<0,001)$. Tanto o peso e o IMC correlacionaram-se positivamente com o somatório dos fatores do teste EBBIT $(r=0,621 ; p<0,001$ e $r=0,645$; $\mathrm{p}<0,001)$ e o somatório da insatisfação com a imagem corporal com o peso $(r=0,659 ; p<0,001$ e $r=0,701$; $p<0,001)$. Considerações finais: a insatisfação com a imagem corporal está presente na população estudada, tanto em escolares adolescentes eutróficos, quanto nos com sobrepeso e/ou obesidade. Quanto aos comportamentos alimentares, indivíduos com sobrepeso e/ou obesidade foram os que mais pontuaram positivamente, contribuindo para a incidência sugestiva de transtornos de origem no comportamento alimentar.

Palavras-chave: Estado Nutricional; Imagem Corporal; Comportamento Alimentar; Transtorno Alimentar.

\section{ABSTRACT}

Objective: associate the nutritional status, the eating behaviors and the body satisfaction of adolescent students of Santa Cruz do Sul, RS. Method: this is a cross-sectional study, accomplished with 54 adolescent students aged between 10 and 12 years $(10,49 \pm 0,50$ years). The nutritional state was evaluated by Body Mass Index (BMI) calculation and eating behaviors through was evaluated the questionnaire Eating Behaviours and Body Image Test (EBBIT). To assess the satisfaction with the body image was used the silhouette scale proposed by Tiggeman and Wilson-Barrett. Results: of the 54 studied adolescents, the majority (53.7\%) presented eutrophy and $61.1 \%$ were dissatisfied for overweight. It was found a significant association between the body image dissatisfaction and the nutritional status $(p=0.001)$. We found a significant difference between the nutritional status and the sum of factors in the EBBIT test $(p<0.001)$ as well as in the score of the sum with body image satisfaction $(p<0.001)$. Both the weight and BMI 
were correlated positively with the sum of factors of EBBIT test ( $r=0.621 ; p<0.001$ and $r=0.645 ; p<0.001)$ and the sum of dissatisfaction with the body image and weight ( $r=0.659 ; p<0.001$ e $r=0.701 ; p<0.001)$. Closing remarks: body image dissatisfaction is present in the studied population, both in eutrophic adolescent students and in those overweight and/or obese. As for the eating behaviors, overweight and/or obese subjects were those who most scored positively, indicating the possible presence of an eating disorder.

Keywords: Nutritional Status; Body Image; Eating Behaviour; Eating Disorder.

\section{INTRODUCÃ̃O}

Os distúrbios relacionados à satisfação com a imagem corporal e comportamentos alimentares geralmente tem início no período da adolescência, no qual são identificados a presença de sintomas relacionados aos transtornos alimentares. ${ }^{1}$ Além disso, a distorção da imagem corporal, é um dos fatores determinantes para o surgimento de transtornos de origem alimentar. ${ }^{2}$

A imagem corporal consiste numa idealização multidimensional, defendida pelas percepções e atitudes que o indivíduo tem em relação ao seu próprio corpo. ${ }^{3}$ É durante a fase escolar que ocorrem as principais mudanças corporais, e muitos dos comportamentos alimentares são direcionados à determinado padrão de beleza, gerando uma insatisfação com a imagem corporal. Devido a essa insatisfação, adolescentes adotam práticas inadequadas de controle de peso, apresentando maior risco de desenvolvimento de transtornos, quando comparados aos adolescentes satisfeitos com a imagem corporal. ${ }^{4}$

Para determinar o estado nutricional, um dos métodos mais utilizados é a classificação do Índice de Massa Corporal IMC, de acordo com a idade do indivíduo, pois este cálculo reflete melhor as mudanças na forma corporal da criança e do adolescente. ${ }^{5}$ Sendo de grande importância, o estado nutricional tem como principal objetivo, verificar o crescimento e a composição corporal de cada indivíduo, visando um desenvolvimento adequado, para futuramente estabelecer atitudes de intervenção, se necessário. ${ }^{6}$

Estudos apontam uma associação entre a insatis fação com imagem corporal em adolescentes do sexo feminino e o IMC fora dos padrões de normalidade para eutrofia. ${ }^{7}$ Neste sentido, meninas tendem a apresentar com mais frequência distorção na percepção da imagem corporal do que os meninos, uma vez que elas tendem a observar alterações na sua imagem corporal, mesmo estando no estado de eutrofia. Não muito diferente, os meninos também apresentam distorção na percepção da imagem corporal, porém, geralmente se consideram satisfeitos, mesmo estando com sobrepeso e/ou obesidade. ${ }^{8}$

Além da insatisfação com a imagem corporal, no período da adolescência são constantes os comportamentos alimentares inadequados, caracterizados pelo consumo irregular de refeições, excesso de lanches, refeições rápidas fora de casa, dietas extremas, hábito de pular refeições, entre outros. A maioria dos adolescentes está ciente da importância da nutrição e de uma dieta saudável, mas há diversas barreiras na escolha dos alimentos, como por exemplo, a influência da família ou pessoas próximas, exposição à mídia, aumento de responsabilidades e falta de tempo para uma refeição em família. ${ }^{9}$ Entretanto, fazem uso de dietas restritivas, além de apresentarem aversões aos alimentos, compulsões e comportamento de purgações, pensamentos obsessivos em relação a calorias e aos alimentos, acessos de raiva por sentir fome, necessidade de procurar alimentos que possam compensar problemas psicológicos, entre outros. ${ }^{10}$

Sendo assim, o objetivo do estudo foi relacionar o estado nutricional, comportamento alimentar e a satisfação corporal de escolares adolescentes de Santa Cruz do Sul, RS.

\section{MÉTODO}

População de estudo, inclusão dos participantes e aspectos éticos

Consiste em um estudo transversal, com escolares adolescentes de ambos os sexos, com idades entre 10 até 12 anos, matriculados nas turmas de $5^{\circ}$ e $6^{\circ}$ ano, de uma escola estadual de educação básica, do município de Santa Cruz do Sul/RS, no mês de abril de 2015.

Inicialmente, foram selecionados para participar do estudo, todos os escolares matriculados nas turmas de $5^{\circ}$ e $6^{\circ}$ ano, de uma escola estadual, do município de Santa Cruz do Sul/RS, que estavam presentes e aceitaram participar do estudo (totalizando 178 adolescentes). Estes, após as devidas orientações e esclarecimentos sobre o estudo, receberam os questionários para a pesquisa, o Termo de Consentimento Livre e Esclarecido (TCLE) a ser assinado por um de seus pais e/ou responsável e o Termo de Assentimento Informado Livre e Esclarecido (TAILE) a ser assinado pelo escolar adolescente. Estes seriam respondidos pelos escolares adolescentes em suas residências, sendo combinadas datas para a retirada dos mesmos e dos termos devidamente assinados.

Do total de termos de TCLE e TAILE, e questionários distribuídos (178), foram excluídos 102 estudantes, pois não assinaram os termos de TCLE e TAILE ou não devolveram o questionário preenchido. Ressalta-se que foram realizadas quatro tentativas na escola para devolução dos questionários, TCLE e TAILE, para obtenção da uma amostra maior. Dos 76 questionários respondidos, mais 19 escolares foram excluídos do estudo devido aos seus questionários não estavam corretamente respondidos ou por desistência de participar do estudo. Além disso, foram excluídos 3 indivíduos que apresentaram estado nutricional para magreza, pois delimitou-se a análise aos escolares adolescentes em situação de eutrofia e excesso de peso, totalizando no final uma amostra de 54 escolares.

A presente pesquisa foi aprovada pelo Comitê de Ética em Pesquisa com Seres Humanos (CEP) da Universidade de Santa Cruz do Sul/RS, sob o parecer $n^{\circ}$ 907.508.

\section{Avaliação do estado nutricional}

Primeiramente, realizou-se a medida da massa corporal, utilizando uma balança de chão da marca Plenna ${ }^{\circledR}$ 
com capacidade máxima para $150 \mathrm{~kg}$. Para a aferição da estatura, foi afixada uma fita métrica em uma parede lisa e sem rodapés, sendo a medida devidamente conferida.

Por conseguinte, avaliou-se o estado nutricional, utilizando o cálculo do IMC (peso/estatura²), classificado posteriormente de acordo com o escore $\mathrm{Z}$ das curvas de crescimento para sexo feminino e masculino de acordo com a idade. A classificação se deu através de: eutrofia ( $\geq$ escore $Z-2$ e $<$ escore $Z+1$ ), sobrepeso ( $\geq$ escore $Z+1$ e $<$ escore $Z+2$ ), obesidade ( $\geq$ escore $Z+2$ e $\leq$ escore $Z+3){ }^{11}$

\section{Determinação do comportamento alimentar}

Para a avaliação dos comportamento alimentar, aplicou-se o questionário Eating Behaviours and Body Image Test (EBBIT), criado por Candy e Fee..$^{12} \mathrm{O}$ questionário é composto por 42 questões em escala Likert, com 4 alternativas de resposta, no qual, cada alternativa traduz o comportamento alimentar com uma pontuação diferente. As respostas têm variação de: a maior parte do tempo/todos os dias, até nunca. A pontuação varia de 0 (nunca) a 3 (a maior parte do tempo/todos os dias). Estas 42 questões, foram divididas em três fatores: fator 1 (insatisfação com a imagem corporal e restrição alimentar - itens 1, 2, 4, 5, $6,7,10,12,15,18,19,21,24,25,27,28,29,32,33$, 37, 38, 41 e 42); fator 2 (comer em excesso - itens 3, 8, $9,13,14,16,20,22,26,30,31,34,35,39$ e 40). Esta escala é útil para a avaliação precoce, com capacidade de rastrear atitudes indicadoras de possíveis distúrbios no comportamento alimentar. Todos estes fatores referem-se à insatisfação com a imagem corporal, comer com restrição e comer em excesso ocasionalmente. Há ainda o fator 3, correspondente aos itens 11, 17, 23 e 36; podendo ser analisado individualmente e apenas utilizado como uma informação sobre o sujeito. ${ }^{13}$

\section{Determinação da satisfação com a imagem corporal}

Para a avaliação da satisfação com a imagem corporal, foi utilizada uma escala de silhuetas proposta por Tiggeman e Wilson-Barrett. ${ }^{14}$ Esta escala é caracterizada por nove silhuetas do sexo feminino e masculino, separadas e numeradas em ordem crescente, com extremos de magreza e gordura, com altura estável. O conjunto de silhuetas foi anexado juntamente ao questionário entregue, no qual, os adolescentes deveriam marcar um " $X$ " na figura que mais se identificavam e circular a figura que mais gostariam de ser (perfil desejável). Para categorizar a satisfação com a imagem corporal, foram utilizados três extratos: satisfeitos com a imagem corporal; insatisfeitos para o excesso de peso e insatisfeitos para a magreza. A pontuação se deu através do cálculo da subtração do número da figura atual pelo número da figura escolhida como desejável; considerando-se satisfeitos aqueles que obtiveram pontuação igual a zero, insatisfeitos com o excesso de peso aqueles que obtiveram pontuação positiva e insatisfeitos para magreza aqueles que obtiveram pontuação negativa. ${ }^{15}$

\section{Análise estatística}

A análise estatística foi realizada no software Statistical Package for Social Sciences (SPSS) versão 20.0 (Chicago, IL) e os gráficos foram preparados no GraphPad Prism versão 5.01 (San Diego, CA). Inicialmente, realizou-se estatística descritiva para as variáveis (idade, peso, estatura, IMC e EBBIT) através de média, desvio padrão, mediana, valores mínimo e máximo. Os dados foram verificados quanto à normalidade e homocedasticidade. Para comparação do estado nutricional e idade, sexo e imagem corporal foi realizado o teste qui-quadrado $\left(X^{2}\right)$. Foram realizadas também, análises de correlação de Pearson entre os dados antropométricos de peso e IMC com imagem corporal e EBBIT. Para as análises comparativas entre os diferentes grupos do estado nutricional, verificado pelo IMC (eutrofia, sobrepeso e obesidade) com imagem corporal e EBBIT, utilizou-se ANOVA One Way, seguida de Post hoc de Tukey. O nível de significância utilizado foi $p<0,05$.

\section{RESULTADOS}

A predominância da amostra foi do sexo feminino $(56,1 \%)$, com média de idade de $10,49 \pm 0,50$ anos. As características da amostra estão descritas na tabela 1.

Avaliando o estado nutricional dos escolares, observou-se uma maior frequência de adolescentes eutróficos $(53,7 \%)$. Quanto à satisfação com a imagem corporal, $61,1 \%$ do total de escolares estavam insatisfeitos para o excesso de peso e 33,3\% estavam satisfeitos com a imagem corporal.

Também verificou-se associação significativa entre a insatisfação com a imagem corporal e o estado nutricional para o excesso de peso $(p=0,001)$, no qual, $88,9 \%$ e $93,7 \%$ respectivamente dos escolares adolescentes respectivamente, com sobrepeso e obesidade estavam insatisfeitos para o excesso de peso (Tabela 2).

Deve-se ressaltar que ao analisar a amostra separadamente por sexo, tanto as meninas, quanto os meninos estavam eutróficos $(53,3 \%$ e 54,2\%, respectivamente). Entretanto, ao verificar a percepção corporal dos escolares adolescentes, $63,3 \%$ das meninas e $58,3 \%$ dos meninos eutróficos, estavam insatisfeitos para o excesso de peso.

Tabela 1 - Análise descritiva das variáveis idade, peso, estatura, IMC, Escore total teste EBBIT da amostra ( $\mathrm{n}=54$ ).

\begin{tabular}{|c|c|c|c|c|c|}
\hline & Média & Desvio Padrão & Mediana & Mínimo & Máximo \\
\hline Idade (anos) & 10,48 & $\pm 0,50$ & 10,00 & 10,00 & 11,00 \\
\hline Peso (kg) & 44,85 & $\pm 9,73$ & 41,95 & 27,70 & 68,20 \\
\hline Estatura (m) & 1,48 & $\pm 0,06$ & 1,48 & 1,35 & 1,64 \\
\hline IMC (kg/m²) & 20,27 & $\pm 3,74$ & 19,11 & 14,40 & 26,98 \\
\hline EBBIT (pontos) & 29,40 & $\pm 16,36$ & 29,50 & 2,00 & 68,00 \\
\hline
\end{tabular}


Tabela 2 - Relação do estado nutricional com idade, sexo e imagem corporal $(n=54)$.

\begin{tabular}{|c|c|c|c|c|c|}
\hline Idade & Eutrofia & Sobrepeso & Obesidade & Total & $\mathbf{p}$ \\
\hline 10 anos & $12(42,9 \%)$ & $6(21,4 \%)$ & $10(35,7 \%)$ & $28(100,0 \%)$ & 0,248 \\
\hline 11 anos & $17(65,4 \%)$ & $3(11,5 \%)$ & $6(23,1 \%)$ & $26(100,0 \%)$ & \\
\hline \multicolumn{6}{|l|}{ Sexo } \\
\hline Feminino & $16(53,3 \%)$ & $4(13,3 \%)$ & $10(33,3 \%)$ & $30(100,0 \%)$ & 0,682 \\
\hline Masculino & $13(54,2 \%)$ & $5(20,8 \%)$ & $6(25,0 \%)$ & $24(100,0 \%)$ & \\
\hline \multicolumn{6}{|l|}{ Imagem corporal } \\
\hline Satisfeito com a imagem corporal & $16(88,9 \%)$ & $1(5,6 \%)$ & $1(5,6 \%)$ & $18(100,0 \%)$ & 0,001 \\
\hline Insatisfeito para a magreza & $3(100,0 \%)$ & $0(0,0 \%)$ & $0(0,0 \%)$ & $3(100,0 \%)$ & \\
\hline Insatisfeito para o excesso de peso & $10(30,3 \%)$ & $8(24,2 \%)$ & $15(45,5 \%)$ & $33(100,0 \%)$ & \\
\hline
\end{tabular}

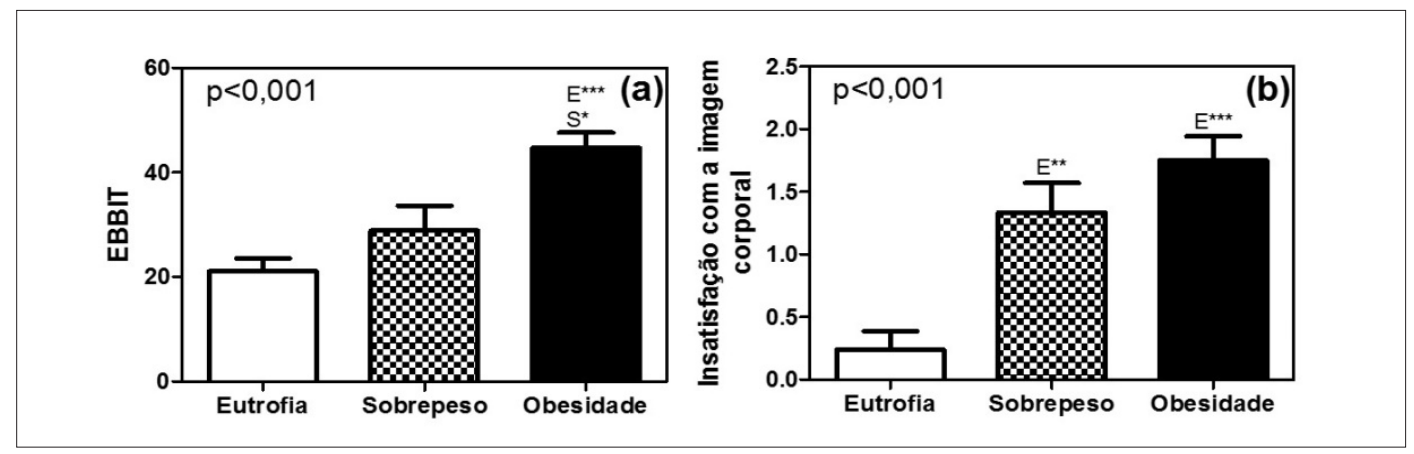

Figura 1 - Comparação entre o estado nutricional com o EBBIT (a) e insatisfação com a imagem corporal (b) de escolares adolescentes com idade entre 10 e 12 anos do sexo feminino e masculino. p: nível de significância de acordo com o teste ANOVA One Way, seguida de Post hoc de Tukey.

Ainda, em relação ao estado nutricional (Figura 1), pode-se observar que os escolares adolescentes com obesidade, obtiveram uma maior pontuação no somatório dos fatores 1 e 2 do teste EBBIT, em relação aos escolares com eutrofia e sobrepeso, de forma significativa $(p<0,001)$. Observou-se ainda que os escolares adolescentes com sobrepeso e obesidade apresentaram maior insatisfação com a imagem corporal para o excesso de peso em relação à eutrofia, de forma significativa $(p<0,001)$.

Considerando o teste EBBIT, verificou-se que os escolares que apresentaram maior peso, bem como maior IMC, apresentaram maior somatório dos fatores 1 e 2 do teste EBBIT ( $r=0,621 ; p<0,001$ e $r=0,645$; $p<0,001)$. Observou-se também uma associação positiva entre o somatório da insatisfação com a imagem corporal com o peso $(\mathrm{r}=0,659 ; p<0,001)$ e o IMC $(\mathrm{r}=0,701 ; p<0,001)$ (Figura 2).

\section{DISCUS5ÃO}

Ao analisar o estado nutricional dos escolares, bem como os parâmetros de satisfação ou insatisfação corporal e os comportamentos alimentares, observou-se que a insatisfação com a imagem corporal para o excesso de peso esteve presente em todos os estados nutricionais, porém predominantemente nos casos de sobrepeso e obesidade.

Nas últimas duas décadas, as pesquisas sobre imagem corporal se intensificaram, principalmente pelas evidências de que a insatisfação com o corpo tem início em idades precoces e é fortemente influenciada
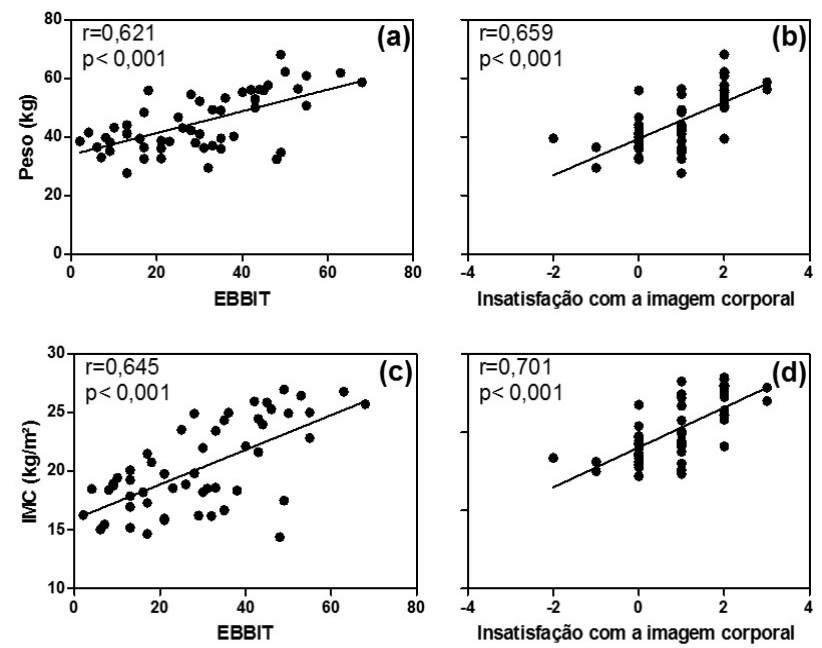

Figura 2 - Associação entre o peso corporal $(\mathrm{kg})$ e IMC $(\mathrm{kg} / \mathrm{cm} 2)$ com o teste EBBIT $(a, c)$ e insatisfação com a imagem corporal (b, d). r e p: coeficiente de correlação e nível de significância, respectivamente, de acordo com o teste de correlação de Pearson.

por aspectos socioculturais, associados com desfechos negativos. ${ }^{16,7} \mathrm{Em}$ adolescentes, de modo geral, a insatisfação foi considerada um fator de risco para presença de humor depressivo, baixa autoestima e intenção suicida, apesar do ajuste para problemas psicológicos e outras variáveis. Meninas no início da adolescência e meninos na adolescência representaram os grupos etários mais vulneráveis à ideação suicida. ${ }^{17,18}$ 
Também, pode-se observar que o sobrepeso e a obesidade possivelmente estariam contribuindo para a insatisfação corporal dos adolescentes estudados, uma vez que se verificou uma associação significativa entre a insatisfação com a imagem corporal e o estado nutricional $(p=0,001)$. Estes resultados também foram observados em outros estudos, nos quais os autores concluem que a insatisfação corporal tende a aumentar conforme os maiores valores de peso e IMC, sendo estes, considerados fortes indicadores contribuintes para a insatisfação corporal. ${ }^{12,19,20,7}$

Entretanto, mesmo em situações de eutrofia, houve a presença de insatisfação para o excesso de peso, indiferentemente do sexo. Através da avaliação do estado nutricional dos escolares, obteve-se uma maior frequência de eutrofia, porém, uma prevalência de insatisfação com a imagem corporal para o excesso de peso de $61,1 \%$. Resultados semelhantes também foram encontrados em um estudo desenvolvido com crianças e adolescentes de 8 a 12 anos de idade, no qual observou-se uma prevalência de $64 \%$ de insatisfação com o corpo em uma amostra com incidência de $26,4 \%$ de sobrepeso e obesidade e $73,6 \%$ de eutrofia. ${ }^{19}$

Durante a puberdade, muitos fatores podem alterar a forma que cada indivíduo enxerga seu corpo, em ambos os sexos, tratando-se de um período de modificações corporais importantes, que precisam passar por um processo de aceitação. ${ }^{21,22}$ Deve-se ressaltar que no presente estudo, tanto as meninas, quanto os meninos estavam eutróficos $(53,3 \%$ e $54,2 \%$, respectivamente). Entretanto, ao verificar a sua percepção corporal, $63,3 \%$ das meninas estavam insatisfeitas para o excesso de peso, corroborando com um estudo desenvolvido no qual verificou-se uma prevalência de $75,8 \%$ de insatisfação com a imagem corporal no sexo feminino com idade entre 11 e 14 anos. ${ }^{23}$ Alves et al.. ${ }^{4}$ verificaram uma prevalência de insatisfação corporal em escolares do sexo feminino com idade entre 10 e 19 anos $(18,8 \%)$, inferior ao do nosso estudo. Já, no estudo realizado por Conti et al., ${ }^{24}$ em adolescentes de ambos os sexos e com idade entre 10 e 14 anos, também pode-se observar uma predominância maior de insatisfação com a imagem corporal entre meninas, quando comparadas aos meninos. ${ }^{24}$

Ao avaliar os comportamentos alimentares, percebeu-se que os escolares adolescentes com sobrepeso e obesidade foram os que mais pontuaram no somatório dos fatores do teste EBBIT. Este resultado pode estar refletindo possíveis indicadores de distúrbios no comportamento alimentar e insatisfação corporal, segundo o teste. ${ }^{14}$

Muitos estudos mostram, que a imagem corporal ideal para o sexo feminino, tanto para adolescentes, quanto para adultas, é predominantemente focado na redução de peso e está associada ao padrão de magreza. ${ }^{25-27}$ Dentre outros fatores, a mídia difunde a ideia de perfeição corporal, onde a magreza simboliza competência, sucesso e atração sexual, enquanto a obesidade representa a preguiça, autopiedade e menor poder de decisão e qualidade de vida. ${ }^{28}$

De acordo com Carvalho et al., ${ }^{1}$ ao avaliar adoles centes do sexo feminino entre 10 e 12 anos, foi encontrada relação direta entre a massa corporal, o IMC e o teste EBBIT, demonstrando que quanto maior a massa corporal e o IMC, maiores serão os escores do teste EBBIT. Desta forma, esses resultados podem indicar um aumento no comportamento alimentar relacionado aos transtornos alimentares. ${ }^{1,14}$

Em outro estudo, desenvolvido com adolescentes de ambos os sexos, com idade entre 10 e 12 anos, observou-se uma diferença significativa ao comparar obesos com adolescentes eutróficos. Os autores verificaram que os adolescentes obesos tiveram maior pontuação nos fatores do teste EBBIT, demonstrando comportamentos alimentares indicativos de comer excessivo e insatisfação com a imagem corporal. ${ }^{29}$

Os resultados obtidos, demonstram que a escala de silhuetas e o teste de comportamentos alimentares e imagem corporal aplicados foram instrumentos úteis para avaliar a população analisada. A partir disso, pode-se identificar a presença de insatisfação corporal em diferentes estados nutricionais, predominantemente em escolares adolescentes do sexo feminino e com estado nutricional de eutrofia, bem como, identificar a população que apresenta transtornos de origem no comportamento alimentar e imagem corporal, no qual predominaram escolares adolescentes com sobrepeso e obesidade.

A partir dos resultados obtidos, conclui-se que a insatisfação com a imagem corporal para o excesso de peso está presente na grande maioria dos escolares adolescentes, não somente no estado nutricional de sobrepeso e obesidade, como esperado, mas também em situação de eutrofia.

Esta forte insatisfação com a imagem corporal demonstra uma percepção alterada que os escolares têm sobre seu próprio corpo, podendo a satisfação corporal ser considerada um problema de saúde pública, devido aos comportamentos que os escolares possam adotar futuramente, por ter essa distorção na percepção de sua própria imagem.

Além disso, pode-se perceber que os indivíduos com sobrepeso e obesidade, as características de comportamentos alimentares inadequados foram predominantes.

Dentre as limitações do estudo, podem-se considerar a perda de indivíduos no processo de seleção da amostra. Trata-se de um tema delicado, desconhecido por muitas famílias, alvo de preconceito ou mesmo desejo de não exposição de problemas, entendeu-se que muitas famílias podem não ter autorizado a participação de seus filhos na pesquisa.

Tudo isso, demonstra a importância de avaliar mais afundo a população estudada, trazer essa discussão para a escola e as famílias, visto que os escolares adolescentes apresentaram elevado índice de insatisfação com a imagem corporal e comportamentos alimentares que podem desencadear transtornos de origem alimentar.

\section{REFERÊNCIAS}

1. Carvalho PHB, Sousa CZ, Cipriani CB, Miranda VPN, Ferreira MEC. Satisfação corporal e comportamentos alimentares em adolescentes do sexo feminino. Coleç Pesqui Educ Fís 2011;10(3):165-72

2. Nunes MA, Olinto MTA, Barros FC, Camey S. Influência da percepção do peso e do índice de massa corporal nos comportamentos alimentares anormais. Rev Bras Psiquiatr 2001:23(1):21-7. 
3. Saur AM, Pasian SR. Satisfação com a imagem corporal em adultos de diferentes pesos corporais. Aval. Psicol 2008;7(2):199-209.

4. Alves E, Vasconcelos FAG, Calvo MCM, Neves J. Prevalência de sintomas de anorexia nervosa e insatisfação com a imagem corporal em adolescentes do sexo feminino do Município de Florianópolis, Santa Catarina, Brasil. Cad Saúde Publ 2008;24(3):503-12. doi: 10.1590/S0102311X2008000300004.

5. Vitolo MR. Nutrição: da gestação ao envelhecimento. $2^{\mathrm{a}}$ ed. Rio de Janeiro: Rubio; 2008.

6. Sigulem DM, Devincenzi MU, Lessa AC. Diagnóstico do estado nutricional da criança e do adolescente. J Pediatr 2000;76(3):275-84.

7. Corseuil MW, Pelegrini A, Beck C, Petroski, EL. Prevalência de insatisfação com a imagem corporal e sua associação com a inadequação nutricional em adolescentes. Rev Educ Fís/UEM 2009;20(1):25-31. doi: 10.4025/reveducfis. v20i1.3496.

8. Branco LM, Hilário MOE, Cintra IP. Percepção e satisfação corporal em adolescentes e a relação com seu estado nutricional. Rev Psiquiatr Clín 2006;33(6):292-96. doi: 10.1590 /S0101 - 60832006000600001

9. Stang J. Nutrição na adolescência. In: Mahan LK, EscottStump S. Krause: Alimentos, Nutrição e Dietoterapia. $12^{\mathrm{a}} \mathrm{ed}$. São Paulo: Roca, 2010. Unidade 19.

10. Dunker K, Timerman F, Alvarenga MS, Philippi ST. Prevenção dos transtornos alimentares e posturas do nutricionista. In: Alvarenga MDS, Scagliusi FB, Philippi ST. Nutrição e transtornos alimentares: Avaliação e tratamento. $1^{\text {a }}$ ed . Barueri: Manole. 2011. Unidade 21

11. World Health Organization - WHO. WHO Child Growth Standards: length/height-forage, weight-for-age, weightfor-length, weight-for-height and body mass index-for-age: methods and development. Geneva: World Health Organization; 2007. [acesso 2015 abr 16]. Disponível em: <http://www. who.int/childgrowth/standards/technical_report/en/>.

12. Candy $\mathrm{C}$, Fee $\mathrm{V}$. The underlying dimensions and psychometric properties of the Eating Behaviours and Body Image Test (EBBIT) for preadolescent girls. J Clin Child Psychol 1998;27(1):117-27.

13. Galindo EMC, Carvalho AMP. Tradução, adaptação e avaliação da consistência interna do Eating Behaviours and Body Image Test para uso com crianças do sexo feminino. Rev Nutr 2007;20(1):47-54.

14. Tiggeman M, Wilson-Barrett E. Children's figure ratings: relationship to self steem and negative stereotyping. Int. J. Eat. Disord 1998;23:83-8. doi: 10.1002/(SICI)1098108X(199801)23:1<83::AID-EAT10>3.0.CO;2-O

15. Santini AP, Kirsten VR. Relação entre o perfil nutricional e a imagem corporal de escolares e adolescentes matriculados em escolas do meio rural da cidade de Santa Maria, RS. Rev. da AMRIGS 2012;56(1):32-7

16. Triches RM, Giugliani ERJ. Insatisfação corporal em escolares de dois municípios da região Sul do Brasil. Rev Nutr 2007;20:11928. doi: 10.1590/S1415- 52732007000200001.

17. Paxton SJ, Sztainer DN, Hannan PJ, Eisenberg ME. Body Dissatisfaction Prospectively Predicts Depressive Mood and Low Self-Esteem in Adolescent Girls and Boys. J Clin Child Adolesc Psychol 2006;35:539-49.

18. Kim DS. Body image dissatisfaction as an important contributor to suicidal ideation in Korean adolescents: gender difference and mediation of parent and peer relationships. $J$ Psychosom Res 2009;66:297-303.

19. Pinheiro J, Jiménez M. Percepção e insatisfação corporal: um estudo em crianças brasileiras. Psico 2010;41(4):510-16.

20. Miranda VPN, Conti MA, Carvalho PHB, Bastos RR, Ferreira MEC. Imagem corporal em diferentes períodos da adolescência. Rev Paul Pediatr 2014;32(1):63-9. doi: 10.1590/S0103-05822014000100011.

21. Bearman SK, Martinez E, Stice E: The skinny on body dissatisfaction: a longitudinal study of adolescent girls and boys. J Youth Adolesc 2006;35:217-29.

22. McCabe MP, Ricciardelli LA: A longitudinal study of pubertal timing and extreme body change behaviors among adolescent boys and girls. Adolescence 2004:39:145-66.

23. Scherer FC, Martins CR, Pelegrini A, Matheus SC, Petroski EL. Imagem corporal em adolescentes: associação com a maturação sexual e sintomas de transtornos alimentares. J Bras Psiquiatr 2010;59(3): 198-202. doi: 10.1590/S004720852010000300005.

24. Conti MA, Frutuoso MFP, Gambardella AMD. Excesso de peso e insatisfação corporal em adolescentes. Rev Nutr 2005;18(4):491-97. doi: 10.1590/\$141552732005000400005.

25. Forestell CA, Humphrey TM, Stewart SH: Involvement of body weight and shape factors in ratings of attractiveness by women: a replication and extension of Tassinary and Hansen. Pers Individ Dif 2004;36:295-305.

26. Smolak L: Body image in children and adolescents: where do we go from here? Body Image 2004;1:15-28.

27. Guendouzi J: 'She's very slim': talking about body-size in allfemale interactions. J Pragmat 2004;36:1635-53.

28. Pereira EF, Graup S, Lopes AS, Borgato AF, Daraonco LSE. Percepção da imagem corporal de crianças e adolescentes com diferentes níveis socio-econômicos na cidade de Florianópolis, Santa Catarina, Brasil. Rev Bras Saúde Matern Infant 2009;9(3):253-62. doi: 10.1590/S151938292009000300004.

29. Carvalho AMP, Cataneo C, Galindo EMC, Malfará CT. Auto conceito e imagem corporal em crianças obesas. Paidéia 2005;15(30):131-9. doi: 10.1590/S0103863X2005000100014. 